\title{
The Case of a Zebra That Was Misdiagnosed as a Horse: Pulmonary Tumor Thrombotic Microangiopathy, a New Paraneoplastic Syndrome, Mimicking PD-1-Induced Pneumonitis
}

\author{
Corey A. Carter ${ }^{a}$ Robert Browning ${ }^{a}$ Bryan T. Oronsky ${ }^{b}$ Jan J. Scicinski ${ }^{b}$ \\ Christina Brzezniak ${ }^{a}$ \\ ${ }^{a}$ Walter Reed National Military Medical Center, Bethesda, Md., and \\ ${ }^{\mathrm{b}}$ EpicentRx, Mountain View, Calif., USA
}

\section{Key Words}

Pulmonary tumor thrombotic microangiopathy - Paraneoplastic syndrome · PD-1-induced pneumonitis

\begin{abstract}
A case report of a 47-year-old woman with triple-negative breast cancer on a clinical trial called PRIMETIME (NCT02518958) who received the anti-PD-1 inhibitor nivolumab and the experimental anticancer agent RRx-001 is presented. Although initially diagnosed and treated for anti-PD-1-induced pneumonitis, clinical and radiological abnormalities triggered further investigation, leading to the diagnosis of pulmonary tumor thrombotic microangiopathy (PTTM). This example highlights the importance of exercising due diligence in determining immune-related adverse events and suggests that PD-1-induced pneumonitis should be a diagnosis of exclusion rather than a diagnosis by default. A case history and review of the literature are presented for PTTM, which we propose to define as a paraneoplastic syndrome.
\end{abstract}


Carter et al.: The Case of a Zebra That Was Misdiagnosed as a Horse: Pulmonary Tumor Thrombotic Microangiopathy, a New Paraneoplastic Syndrome, Mimicking PD-1-Induced Pneumonitis

\section{Introduction}

According to the well-known expression 'When you hear hoofbeats, think of horses not zebras', the art of medicine is based on soundness: the higher the pretest probability, the sounder the diagnosis. The problem with this medical aphorism is that it actively encourages the clinician to turn a deaf ear (and a blind eye) to the possibility of lesser known and, therefore, more easily overlooked disease states that mimic or 'sound like' the more expected 'horse' diagnosis. This report presents a case in point: a 47-year-old woman with triplenegative breast cancer on a clinical trial called PRIMETIME (NCT02518958) who received the anti-PD-1 inhibitor nivolumab and the experimental anticancer agent RRx-001 for 18 weeks; initially treated for pneumonitis, an 'expected' autoimmune complication of nivolumab, based on the development of dyspnea and CT abnormalities. The overall clinical picture, nevertheless, was atypical, which prompted the investigating team to aggressively pursue alternate possibilities, ultimately leading to the correct diagnosis: pulmonary tumor thrombotic microangiopathy or PTTM.

This example highlights the importance of exercising due diligence and not automatically jumping to conclusions with regard to the diagnosis of immune-related adverse events (irAEs) such as pneumonitis during treatment with PD-1 or CTLA-4 inhibitors. Analogous to another pulmonary medical aphorism, 'all that wheezes is not asthma', the differential diagnosis for breathlessness in the context of immune checkpoint inhibition is broader than only pneumonitis and should involve a systematic investigation for other etiologies, including the rare and rapidly progressive disorder PTTM. A case history and review of the literature are presented for PTTM, which we propose to define as a paraneoplastic syndrome (PNS). In addition, a potential treatment option based on its pathophysiology is discussed.

The goal of cytotoxic T-lymphocyte antigen-4 (CTLA-4) and programmed death-1 (PD1) pathway blockade, including nivolumab approved for the treatment of melanoma, squamous-cell lung cancer [1] and renal cell carcinoma [2], is to overcome the T-cell suppression mediated by these inhibitory receptors (fig. 1); a potential side effect of revving up the immune system to attack malignant tumors is the breaking of self-tolerance and the induction of irAEs [3], which include rash, colitis, hepatotoxicities, endocrinopathies, and interstitial pneumonitis [4].

As the most serious irAE, reportedly responsible for 5 total fatalities across the spectrum of nivolumab-treated patients [5], the incidence of pneumonitis increased from $3.4 \%$ on a melanoma trial to $6 \%$ on a NSCLC clinical trial, according to a recent Bristol Myers Squibb press release [6]; this increase in incidence should raise the suspicion that increased awareness of and, consequently, narrowed focus on pneumonitis by oncologists has resulted in erroneous overdiagnosis. The clinical manifestations of pneumonitis are protean and include fever, chills, malaise, cough, chest tightness, hypoxia, and dyspnea, while the nonspecific radiological characteristics [7] of ground glass opacities (i.e., lung opacities that do not obscure the associated vessels), consolidations (i.e., lung opacities that do obscure the associated vessels), and effusions also overlap with multiple other disease entities including acute respiratory distress syndrome, pneumonia, pulmonary embolism (PE), congestive heart failure, and the subject of this case report, PTTM.

PTTM is a rare and possibly underdiagnosed [8] extrapulmonary sequella of metastatic cancer, specifically adenocarcinomas [9], formally described in 1990 by von Herbay et al. [10] that presents as acute cor pulmonale, a maladaptive response to pulmonary hypertension [11], resulting in dyspnea and hypoxemia as well as ground-glass opacity (or diffuse consolidation) and pulmonary edema on CT $[12,13]$. The available literature on PTTM is 
Carter et al.: The Case of a Zebra That Was Misdiagnosed as a Horse: Pulmonary Tumor Thrombotic Microangiopathy, a New Paraneoplastic Syndrome, Mimicking PD-1-Induced Pneumonitis

sparse, existing mostly as case reports or small case series from Japan, with a lack of higherorder treatment studies. Adenocarcinomas, and gastric cancer in particular [14], have been linked with PTTM in these Japanese case reports, which is not surprising, given the high incidence rate of gastric cancer in Japan.

The etiologic mechanism of PTTM is related to the intravasation of circulating tumor cells in the pulmonary vasculature; these circulating tumor cells release a plethora of vascular remodeling factors [15] including vascular endothelial growth factor (VEGF), fibroblast growth factor, osteopontin [16], and platelet derived growth factor (PDGF) associated with abnormal endothelial proliferation, the local activation of the coagulation cascade, and the development of pulmonary hypertension from resultant stenosis of the pulmonary capillaries and arterioles (fig. 2).

In the absence of gold standard surgical biopsy, PTTM, which is only rarely diagnosed antemortem, due to a nearly uniform fatality rate [17], (almost all reported patients have died within 2 weeks of dyspnea onset), may be suspected in cancer patients ruled out for PE who develop acute or subacute right-sided heart failure, pulmonary hypertension, and abnormal coagulation parameters.

Our nivolumab-treated patient with metastatic triple-negative breast cancer who is the subject of this case report recapitulated these clinical and laboratory abnormalities almost 'to a $\mathrm{T}$ ', including, unfortunately, the time interval between onset of first symptoms and death. While the term 'textbook example' is not applicable in the context of PTTM, since no textbooks on it have been written (only case reports), this patient's clinical course and trajectory were indeed 'textbook' for PTTM, even though a default diagnosis of nivolumabinduced pneumonitis was initially made. However, in light of the atypical presentation, discussed below, and findings that did not quite 'add up', the team, suspecting that pneumonitis was a red herring, decided to trust their intuition, and pursued a further work-up, which ultimately led to the diagnosis of PTTM.

\section{Case Report}

A 47-year-old female with rapidly progressive refractory triple-negative metastatic breast cancer, metastatic to the lungs, was treated at Walter Reed on a clinical trial called PRIMETIME (NCT02518958) where she received the anti-PD-1 inhibitor nivolumab, 3 $\mathrm{mg} / \mathrm{kg}$, every other week in combination with weekly RRx-001, an experimental epiimmunologic agent. During her week 18 infusion on December 3, 2015, she developed fever $\left(101^{\circ} \mathrm{F}, 38.3^{\circ} \mathrm{C}\right)$, headache, palpitations, and diaphoresis; the presence of fever prompted a sepsis work-up consisting of complete blood count, routine blood culture, urinalysis, and chest X-ray in the hospital to determine the source of the fever and rule out an infection.

Examination revealed tachycardia to $124 \mathrm{bpm}$. A spiral CT scan was negative for PE. The remainder of the physical exam was unremarkable. ECG recordings revealed only sinus tachycardia. Septic work-up was negative. Glycemia was in the normal range $(120 \mathrm{mg} / \mathrm{dl})$. Her labs were otherwise significant only for anemia (hemoglobin $9.4 \mathrm{~g} / \mathrm{dl}$ and hematocrit $30.6 \%)$ and a normal leukocyte count $\left(5.8 \times 10^{3} / \mu \mathrm{l}\right)$ with a high percentage of segmented neutrophils on the differential (normal 43-73\%) and no bandemia. As part of the work-up for sinus tachycardia, thyroid function tests were performed but thyroid-stimulating hormone (TSH) and free T4 were within normal limits.

Her symptoms progressed to dyspnea and hypoxia the next day (on December 4, 2015) after administration of isotonic normal saline to restore volume. Chest CT, which had been 
Carter et al.: The Case of a Zebra That Was Misdiagnosed as a Horse: Pulmonary Tumor Thrombotic Microangiopathy, a New Paraneoplastic Syndrome, Mimicking PD-1-Induced Pneumonitis

clear on admission, revealed interlobular septal thickening, diffuse ground-glass attenuation, and bilateral effusions (fig. 3).

A shortened list of differential diagnoses included atypical viral or bacterial pneumonia, cardiogenic pulmonary edema (since the patient had received doxorubicin in the past), and treatment-induced pneumonitis. On that basis, i.v. fluids were withheld, the patient was discontinued from the trial and combination treatment with broad-spectrum empiric antibiotics (vancomycin, Zosyn and levaquin) and $1 \mathrm{mg} / \mathrm{kg}$ prednisone was started. However, the antibiotics and corticosteroids were withdrawn a week later due to worsening dyspnea and hypoxia. A ventilation-perfusion scan was performed on December 14, 2015 and read as low probability for PE (no perfusion defects).

To identify the cause of the edema, a transthoracic echocardiography was ordered, which revealed a normal left ventricular ejection fraction with right ventricular dilatation and severely reduced right ventricular function secondary to pulmonary hypertension (a pressure $>50 \mathrm{~mm} \mathrm{Hg}$ is generally accepted as severe [18] and her estimated right ventricular systolic pressure exceeded $65 \mathrm{~mm} \mathrm{Hg}$ ). proBNP was also drawn; it was elevated at 3,226.0 $\mathrm{pg} / \mathrm{ml}$, indicative of heart failure (5-125 nl range). Cardiac troponins were not elevated.

The presence of acute cor pulmonale pointed away from pneumonitis and strongly suggested three rare possibilities: (1) occlusion of the pulmonary vessels from microscopic tumor emboli (also known as pulmonary tumor embolism), leading to pulmonary arterial hypertension (PAH); (2) PTTM, a related but even rarer pulmonary vasculopathy, associated with adenocarcinomas of the stomach, pancreas, breast, lung, and liver, in which minute tumor emboli in the peripheral pulmonary arteries damage the vascular endothelium, leading to accelerated coagulation [12] and $\mathrm{PAH}$, and (3) pulmonary venous occlusive disease, the venous form of PAH $[19,20]$, due to fibrous occlusion of the post-capillary vessels, which may or may not be associated with an autoimmune process [10] (the association is only anecdotal). The pulmonary hypertension was managed with diuretics (furosemide $40 \mathrm{mg}$ i.v.), bosentan $62.5 \mathrm{mg}$, and oxygen (2 liters by nasal cannula) as well as intravenous epoprostenol, norepinephrine, and dobutamine; however, no improvement was observed.

Since a lung biopsy carried too much risk, the patient was catheterized; pulmonary artery catheter-derived blood samples were negative for the presence of tumor emboli. Blood was also drawn for the measurement of coagulation parameters, since PTTM is associated with coagulopathy. The results were as follows: elevated D-dimer (or serum fibrin degradation products) of $1.04 \mu \mathrm{g} / \mathrm{ml}$ (normal $<0.5 \mu \mathrm{g} / \mathrm{ml}$ ) and a prolonged prothrombin time of 16.5 $\mathrm{s}$ (normal 11-14 s). Platelets were never outside of the normal range. The partial thromboplastin time and thrombin time were both prolonged at $67.2 \mathrm{~s}$ (normal 25-35 s) and $48 \mathrm{~s}$ (normal 14-20 s), respectively, due to treatment with a heparin GTT for potential venous clots.

In the setting of adenocarcinoma, pulmonary hypertension, and a negative CT and ventilation-perfusion scan for PE, these coagulation abnormalities were highly suggestive of a PTTM diagnosis. PTTM is distinct from simple embolic obstruction because it is characterized by (1) the systemic activation of coagulation with the generation of intravascular fibrin and the consumption of procoagulants, leading to a disseminated intravascular coagulationlike picture, present in this case and (2) remodeling of the pulmonary vasculature [21] due to expression of VEGF and PDGF from embolic tumor cells (see fig. 2) [22].

Based on the proposed involvement of VEGF and PDGF in the pathogenesis of PTTM, the primary investigator planned to treat the patient with sunitinib, which dually inhibits VEGF and PDGF pathways; however, before she could start on treatment, sadly the patient expired. 
Carter et al.: The Case of a Zebra That Was Misdiagnosed as a Horse: Pulmonary Tumor Thrombotic Microangiopathy, a New Paraneoplastic Syndrome, Mimicking PD-1-Induced Pneumonitis

\section{Conclusions}

The observations in this case study strongly suggest that PD-1-induced pneumonitis should be a diagnosis of exclusion rather than a diagnosis by default, requiring a thorough work-up to rule out conditions that may mimic it, including PE, atypical pneumonia, pulmonary venous occlusive disease, congestive heart failure, and PTTM. In the case of this acutely dyspneic patient, who initially received a 'pneumonitis by default' diagnosis, PTTM was only identified when her shortness of breath deteriorated despite treatment with high dose steroids, alerting the principal investigator to the possibility of heart failure, which led to further investigation.

The treating oncologist must be alert to the development or presence of PNS, defined as a 'pathology caused by tumor cells, which systematically produce a large amount of hormones, growth factors, cytokines and a variety of specific symptoms' [23]. These include: Cushing syndrome, carcinoid syndrome, dermatomyositis-polymyositis, myasthenia gravis and syndrome of inappropriate antidiuretic hormone [24]. To this list, we would add PTTM, since its pathogenesis is related to the local release of mitogenic factors associated with vascular remodeling and coagulopathy. As a heretofore unrecognized PNS, PTTM requires background knowledge and a high index of clinical suspicion. In the absence of a biopsy, echocardiographic studies and coagulation tests are the diagnostic tests of choice.

Even though the case under discussion was refractory to standard therapies and the patient died before sunitinib, the multitargeted tyrosine kinase selective for VEGF and PDGF receptors, could be started, it is reasonable to assume that early diagnosis and treatment would have resulted in a better outcome.

\section{Statement of Ethics}

The patient described in this case report has given his informed consent as part of the PRIMETIME clinical study (NCT02518958). This study protocol has been approved by the Walter Reed National Military Medical Center Institutional Review Board.

\section{Disclosure Statement}

B.T.O. and J.J.S. are employees of EpicentRx, Inc. EpicentRx, Inc. provided funding for the study. The remaining authors declare that they have no competing interests.

\section{References}

1 http://packageinserts.bms.com/pi/pi_opdivo.pdf (accessed December 28, 2015).

-2 Motzer RJ, Escudier B, McDermott DF, et al: Nivolumab versus everolimus in advanced renal-cell carcinoma. N Engl J Med 2015;373:1803-1813.

3 Di Giacomo AM, Biagioli M, Maio M: The emerging toxicity profiles of anti-CTLA-4 antibodies across clinical indications. Semin Oncol 2010;37:499-507.

4 Weber JS, Yang JC, Atkins MB, Disis ML: Toxicities of immunotherapy for the practitioner. J Clin Oncol 2015;33:2092-2099.

-5 Topalian SL, Sznol M, McDermott DF, et al: Survival, durable tumor remission, and long-term safety in patients with advanced melanoma receiving nivolumab. J Clin Oncol 2014;32:1020-1030.

6 http://news.bms.com/press-release/follow-data-two-pivotal-opdivo-nivolumab-trials-demonstratessustained-survival-result (accessed December 28, 2015). 
Carter et al.: The Case of a Zebra That Was Misdiagnosed as a Horse: Pulmonary Tumor Thrombotic Microangiopathy, a New Paraneoplastic Syndrome, Mimicking PD-1-Induced Pneumonitis

7 Nishino M, Sholl LM, Hodi FS, Hatabu H, Ramaiya NH: Anti-PD-1-related pneumonitis during cancer immunotherapy. N Engl J Med 2015;373:288-290.

8 Godbole R, Ghatol A, Betancourt J, Sacoolidge J, Kamangar N: Pulmonary tumor thrombotic microangiopathy: clinical, radiologic, and histologic correlation. J Clin Imaging Sci 2015;5:44.

$>9$ Shih HM, Lin CC, Shiao YW: Pulmonary tumor thrombotic microangiopathy. Am J Emerg Med 2011;29:241.e3-241.e4.

10 von Herbay A, Illes A, Waldherr R, Otto HF: Pulmonary tumor thrombotic microangiopathy with pulmonary hypertension. Cancer 1990;66:587-592.

11 Rabinovitch M: Pathobiology of pulmonary hypertension. Annu Rev Pathol 2007;2:369-399.

-12 Uruga H, Fujii T, Kurosaki A, Hanada S, Takaya H, Miyamoto A, Morokawa N, Homma S, Kishi K: Pulmonary tumor thrombotic microangiopathy: a clinical analysis of 30 autopsy cases. Intern Med 2013;52:1317-1323.

-13 Kitamura A, Nishimura N, Jinta T, Suda R, Yamano Y, Ishikawa G, Tomishima Y, Hamaoka T, Suzuki K, Chohnabayashi N: A case of pulmonary tumor thrombotic microangiopathy diagnosed by transbronchial lung biopsy and treated with chemotherapy and long-term oxygen and anticoagulation therapies. Case Rep Pulmonol 2013;2013:259080.

14 Chinen K, Tokuda Y, Fujiwara M, Fujioka Y: Pulmonary tumor thrombotic microangiopathy in patients with gastric carcinoma: an analysis of 6 autopsy cases and review of the literature. Pathol Res Pract 2010;206:682-689.

15 Chinen K, Kazumoto T, Ohkura Y, Matsubara 0, Tsuchiya E: Pulmonary tumor thrombotic microangiopathy caused by a gastric carcinoma expressing vascular endothelial growth factor and tissue factor. Pathol Int 2005;55:27-31.

16 Takahashi F, Kumasaka T, Nagaoka T, et al: Osteopontin expression in pulmonary tumor thrombotic microangiopathy caused by gastric carcinoma. Pathol Int 2009;59:752-756.

17 Sato Y, Marutsuka K, Asada Y, Yamada M, Setoguchi T, Sumiyoshi A: Pulmonary tumor thrombotic microangiopathy. Pathol Int 1995;45:436-440.

18 Berger M, Haimowitz A, Van Tosh A, Berdoff RL, Goldberg E: Quantitative assessment of pulmonary hypertension in patients with tricuspid regurgitation using continuous wave Doppler ultrasound. J Am Coll Cardiol 1985;6:359-365.

Weisser K, Wyler F, Gloor F: Pulmonary veno-occlusive disease. Arch Dis Child 1967;42:322-327.

20 Rosenthal A, Vawter G, Wagenvoort CA: Intrapulmonary veno-occlusive disease. Am J Cardiol 1973;31:7883.

21 Kumar N, Price LC, Montero MA, Dimopoulos K, Wells AU, Wort SJ: Pulmonary tumour thrombotic microangiopathy: unclassifiable pulmonary hypertension? Eur Respir J 2015;46:1214-1217.

22 Demirag F, Cakir E, Yazici U, Tastepe I: Pulmonary tumor thrombotic microangiopathy from metastatic epithelioid angiosarcoma. J Thorac Dis 2013;5:E107-E111.

23 Cui T, Hurtig M, Elgue G, et al: Paraneoplastic antigen Ma2 autoantibodies as specific blood biomarkers for detection of early recurrence of small intestine neuroendocrine tumors. PLoS One 2010;5:e16010.

24 Pelosof LC, Gerber DE: Paraneoplastic syndromes: an approach to diagnosis and treatment. Mayo Clin Proc 2010;85:838-854. 


\section{Case Reports in Oncology}

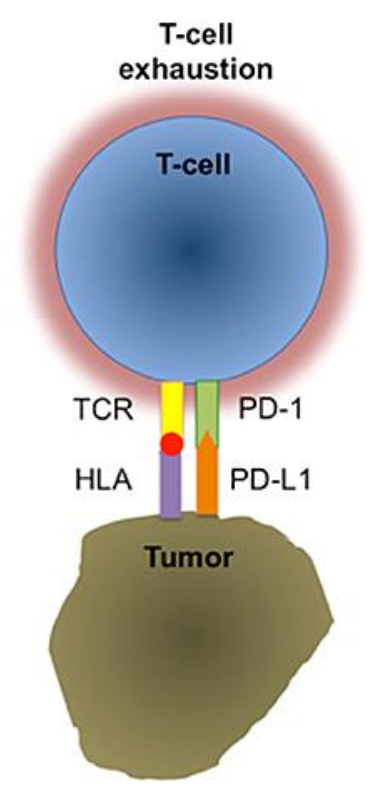

\begin{tabular}{l|l}
\hline Case Rep Oncol 2016;9:68-75 \\
\hline DOI: $10.1159 / 000443723$ & $\begin{array}{l}\text { (c) } 2016 \text { The Author(s). Published by S. Karger AG, Basel } \\
\text { www.karger.com/cro }\end{array}$ \\
\hline
\end{tabular}

Carter et al.: The Case of a Zebra That Was Misdiagnosed as a Horse: Pulmonary Tumor Thrombotic Microangiopathy, a New Paraneoplastic Syndrome, Mimicking PD-1-Induced Pneumonitis

Fig. 1. PD-1 is a cell-surface receptor of the CD28 superfamily that triggers inhibitory pathways to attenuate $\mathrm{T}$-cell responses and promote immune suppression. The PD-1 antibody blocks the interaction between PD-1 and its ligands PD-L1 and PD-L2 (not shown).

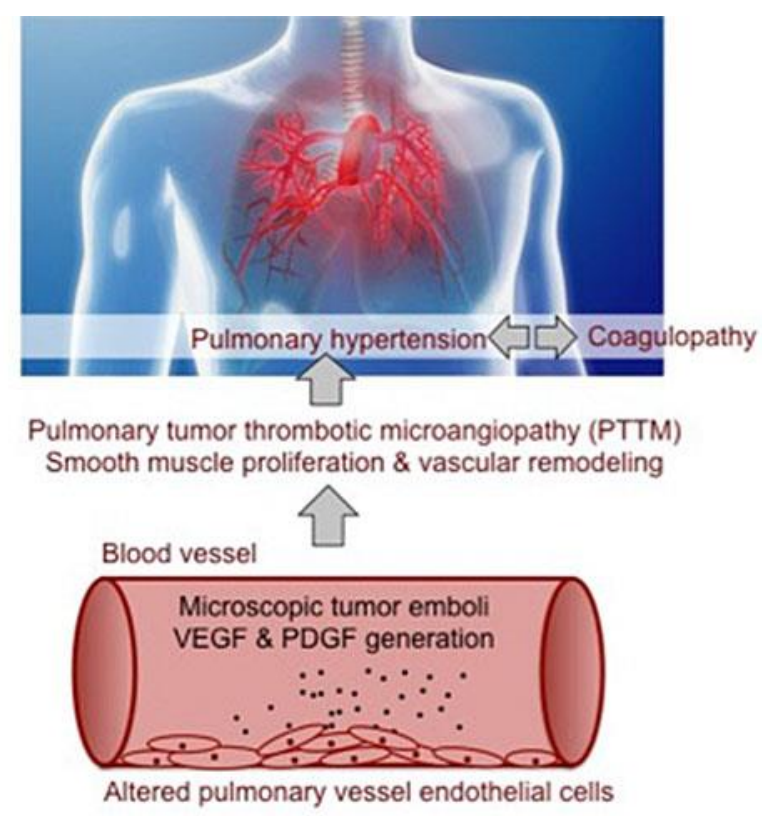

Fig. 2. Proposed mechanism of PTTM. Microscopic tumor emboli due to disease progression release cytokines and growth factors (e.g., VEGF and PDGF) leading to coagulopathy and vascular remodeling. The latter is responsible for pulmonary hypertension. The involvement of VEGF and PDGF justifies or supports treatment with a tyrosine kinase inhibitor like sunitinib that inhibits VEGF and PDGF pathways. 


\section{Case Reports in Oncology}

\begin{tabular}{l}
\hline Case Rep Oncol 2016;9:68-75 \\
\hline DOI: $10.1159 / 000443723$ \\
\hline
\end{tabular}

Carter et al.: The Case of a Zebra That Was Misdiagnosed as a Horse: Pulmonary Tumor Thrombotic Microangiopathy, a New Paraneoplastic Syndrome, Mimicking PD-1-Induced Pneumonitis

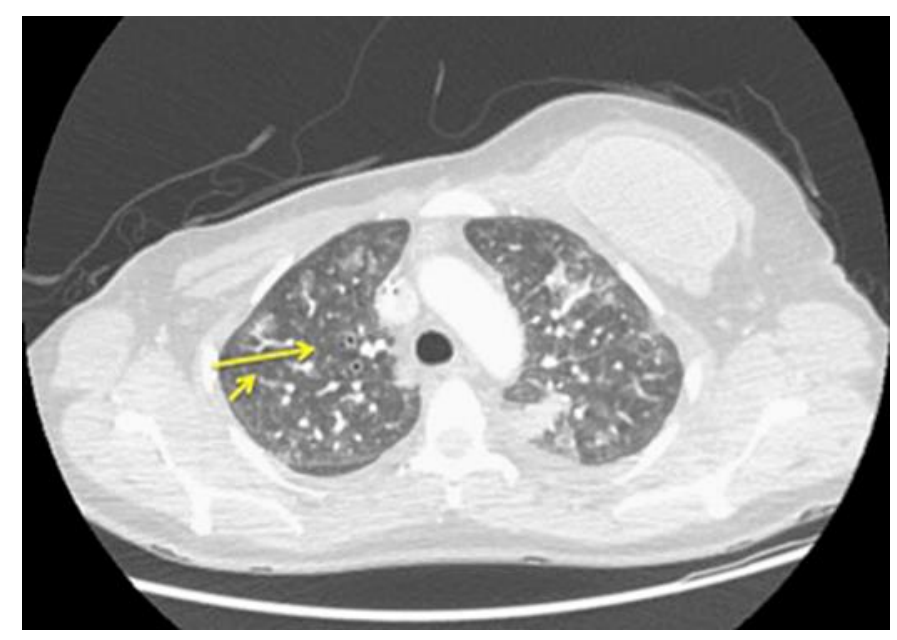

Fig. 3. CT of the lungs demonstrates diffuse ground-glass attenuation, bilateral effusions, and scattered pulmonary nodules. The yellow arrows show prominent interlobular septal thickening. 